\title{
Vocal Cord Paralysis Secondary to Carotid Artery Dissection: A Case Report
}

\author{
Ethel Nkechi Chime ${ }^{1,2 *}$, Peter Ekpunobi Chime ${ }^{3}$, John C. Eze ${ }^{4}$ \\ ${ }^{1}$ Department of Otorhinolaryngology, University of Nigeria Teaching Hospital, Ituku-Ozalla, Enugu, Nigeria \\ ${ }^{2}$ Department of Otorhinolaryngology, Enugu State University of Science and Technology Teaching Hospital, Parklane, Enugu, \\ Nigeria \\ ${ }^{3}$ Department of Medicine, Enugu State University of Science and Technology Teaching Hospital, Parklane, Enugu, Nigeria \\ ${ }^{4}$ Cardiothoracic Unit, Department of Surgery, University of Nigeria, Ituku-Ozalla Campus, Ituku-Ozalla, Enugu State, Nigeria \\ Email: ^ethel.chime@unn.edu.ng, pe4chyme@yahoo.com, johnc.eze@unn.edu.ng
}

How to cite this paper: Chime, E.N., Chime, P.E. and Eze, J.C. (2022) Vocal Cord Paralysis Secondary to Carotid Artery Dissection: A Case Report. Journal of Biosciences and Medicines, 10, 8-12. https://doi.org/10.4236/jbm.2022.103002

Received: October 19, 2021

Accepted: February 28, 2022

Published: March 3, 2022

Copyright (c) 2022 by author(s) and Scientific Research Publishing Inc. This work is licensed under the Creative Commons Attribution International License (CC BY 4.0).

http://creativecommons.org/licenses/by/4.0/

\begin{abstract}
Background and Objective: Vocal cord paralysis results in impairment of breathing and/or speech. One of the causes of vocal cord paralysis is the disruption of vagus nerve innervation to the vocal cords by the mass effect of a neighbouring structure. We report a rare case of vocal cord paralysis secondary to internal carotid artery dissection. Method: The diagnosis was based on clinical history, physical examination and imaging studies. Literature review was done. Case Report: This was a 53-year-old female with a history of unremitting, progressive hoarseness and mild dysphagia to liquid, who was clinically found to have impaired left vocal cord mobility, a left-sided pulsatile neck mass and left carotid artery dissection based on imaging studies. Symptoms abated after conservative treatment with Aspirin and she has remained symptom free since two years of follow-up. Conclusion: Vocal cord paralysis can be a consequence of carotid artery dissection causing mass effect on the vagus nerve. Thus, carotid artery dissection should not be forgotten as a possible cause of vocal cord paralysis in some cases of vocal cord paralysis of uncertain etiology. Treatment with anti-platelet drug can bring about resolution of symptoms and return of vocal cord mobility.
\end{abstract}

\section{Keywords}

Vocal Cord Paralysis, Internal Carotid Artery Dissection/Aneurysm

\section{Introduction}

Vocal cord paralysis (VCP) is the inability of one or both vocal folds to open or close properly with a consequent impairment of breathing and/or speech. The 
paralysis is commonly due to disruption in the innervation (via the vagus nerve) of the vocal cords. The disruption in innervation can be due to but not limited to mass effect on the nerve, neoplastic invasion, nerve transection or thermal injury [1]. Epidemiologically, internal carotid artery (ICA) dissection ranges from 2.5 to 3 per 100,000 [2] and accounts for about $16 \%$ of all causes of vocal cord paralysis [3], occurs more in males than females in the ratio of about 3:2 and occurs mostly in the sixth decade of life [4]. VCP may present clinically as hoarseness, dyspnea or aspiration.

We report a rare case of VCP secondary to ICA dissection and the treatment outcome.

\section{Method}

The patient was seen as an out-patient. Otolaryngologist, Cardiologist, Radiologist and Cardiothoracic Surgeon were all involved in evaluating the patient. The diagnosis of vocal cord paralysis was based on clinical history, physical examination findings and imaging studies. She was treated with an anti-platelet agent and followed up three monthly for 24 months. Literature review was done.

\section{Case Report}

A 53-year old female presented to the Otolaryngology Clinic of Annunciation Specialist Hospital, Emene, Enugu, Nigeria in November, 2018, with progressive change in voice of 3-month duration and occasional mild dysphagia mostly to liquid. There were no other laryngologic symptoms such as difficulty in breathing, aspiration, regurgitation, odynophagia or foreign body sensation in the throat. No nasal or otologic symptoms were complained of. She had left mastectomy sequel to breast cancer five years prior to presentation and had adjuvant chemoradiation.

General examination revealed a middle-aged woman in no obvious respiratory distress, anicteric, not pale, not dehydrated, no finger clubbing and no palpably enlarged peripheral lymph nodes.

Throat examination showed that she had a breathy voice, no stridor. Examination via flexible fiber-optic nasopharyngoscope showed sluggish movement [slow adduction] of the left vocal cord but there was no endolaryngeal mass. There were no remarkable findings in the oral cavity, oropharynx, and hypopharynx. Neck examination showed a mass located on the left side of the middle third of the neck, in the carotid angle, a subdivision of anterior triangle of the neck. The mass was soft, pulsatile, measured approximately $4 \mathrm{~cm}$ by $3 \mathrm{~cm}$, not tender, not attached to underlying or overlying structures and there was no differential warmth. Chest examination revealed a healed scar of Patey's mastectomy. There were no remarkable findings in the other systems.

$\mathrm{X}$-ray soft tissue neck showed an opaque mass on the middle third of the left side of the neck.

Ultrasound scan (USS) of the neck showed a dissecting aneurysm of the left 
internal carotid artery just about $22 \mathrm{~mm}$ above the left common carotid artery bifurcation. Full blood count, coagulation profile, serum electrolytes, urea and creatinine were all within the reference ranges.

The patient was referred to a Cardiothoracic/Vascular surgeon who, after review, placed her on low dose Aspirin for eight weeks. Within six weeks of treatment with Aspirin, the clinical symptoms abated. A repeat flexible fiber-optic examination after 6 weeks of commencing aspirin showed complete recovery of the hitherto paralyzed left vocal cord and resolution of symptoms of hoarseness and dysphagia. Patient is being followed up 3 monthly by both the Otolaryngologist and the Cardiothoracic Surgeon and has remained symptom free.

\section{Discussion}

Vocal cord paralysis sequel to ICA dissection is a rare clinical entity. Vocal cord paralysis can occur congenitally, secondary to extra-laryngeal tumours, surgical trauma like post-thyroidectomy vocal cord paralysis or from neurologic disorders.

This is the first case of vocal cord paralysis secondary to ICA dissection being reported from this health facility. There is paucity of data of this clinical entity in our environment. ICA dissection occurs more commonly from the sixth decade of life. The index patient is 53 years (sixth decade). ICA dissection occurs more in males than females in the ratio of 3:2 [4].

ICA dissection can be caused by any of these entities: congenital connective tissue defects, fibromuscular dysplasia, type-IV Ehlers-Danlos syndrome, Marfan syndrome, ICA redundancy, idiopathic regressing arteriopathy, hypertension and $\alpha 1$-antitrypsin deficiency [5] [6]. The vagus nerve innervates the vocal cords via the recurrent laryngeal nerves. The proximity of the vagus nerve (during its course in the neck) to the cervical portion of the ICA makes the vagus nerve vulnerable to mass effect from pressure of dissecting or aneurysmal ICA with subsequent symptoms of vocal cord paralysis with hoarseness being one of such symptoms.

Treatment of ICA dissection can be conservative as in the index case with good result or use of any of these modalities: balloon angioplasty, distal thrombectomy, ligation of the distal carotid artery, or external-internal carotid bypass [7] [8]. These invasive procedures are recommended for patients who present with recurrent ischemic stroke or for those who are not responsive to conservative therapy [9].

Prognosis of ICA dissection is encouraging. In $23 \%$ to $85 \%$ of the cases, recovery is either fully or nearly fully attained [10] [11].

This patient has been followed up for 24 months and has remained symptom free after completing Aspirin therapy. This is similar to the findings in the cases reported by Nguyeng T, et al. [7]. The rationale for treatment with anti-platelet or anti-thrombotic drugs is that infarcts secondary to ICA dissections are thromboembolic rather than hemodynamic in nature. Thus, they allow for a 
high rate of recanalization of the artery within the first $2-3$ months and for the prevention of ischemic complications [12].

\section{Conclusion and Recommendation}

Internal carotid artery dissection should not be forgotten as a possible cause of vocal cord paralysis in some cases of vocal cord paralysis of uncertain etiology. This is especially so in patients who have the risk factors for the development of carotid artery aneurysm or dissection. Conservative treatment of ICA dissection can suffice in most cases with some exceptions that may need various degrees of surgical procedures or other interventions.

\section{Conflicts of Interest}

The authors declare no conflicts of interest regarding the publication of this paper.

\section{References}

[1] Nguyen, T.T.J., et al. (2013) Vocal Cord Paralysis Secondary to Spontaneous Internal Carotid Dissection: Case Report and Systematic Review of the Literature. Journal of Otolaryngology - Head and Neck Surgery, 42, 34. https://doi.org/10.1186/1916-0216-42-34

[2] Schievink, W.I., Mokri, B. and Whisnant, J.P. (1993) Internal Carotid Artery Dissection in a Community. Rochester, Minnesota, 1987-1992. Stroke, 24, 1678-1680. https://doi.org/10.1161/01.STR.24.11.1678

[3] Mokri, B., Silbert, P.L., Schievink, W.I. and Piepgras, D.G. (1996) Cranial Nerve Palsy in Spontaneous Dissection of the Extracranial Internal Carotid Artery. Neurology, 46, 356-359. https://doi.org/10.1212/WNL.46.2.356

[4] Kearsley, J. (1981) Vocal Cord Paralysis (VCP): An Aetiologic Review of 100 Cases over 20 Years. Australian and New Zealand Journal of Medicine, 11, 663-666. https://doi.org/10.1111/j.1445-5994.1981.tb03543.x

[5] Mokri, B., Houser, O. and Sundt, T. (1977) Idiopathic Regressing Arteriopathy. Annals of Neurology, 2, 466-472. https://doi.org/10.1002/ana.410020605

[6] Schievink, W., Prakash, U., Piepgras, D. and Mokri, B. (1994) Alpha 1-Antitrypsin Deficiency in Intracranial Aneurysms and Cervical Artery Dissection. Lancet, 343, 452-453. https://doi.org/10.1016/S0140-6736(94)92693-X

[7] Bejjani, G.K., Monsein, L.H. and Laird, J.R. (1999) Treatment of Symptomatic Cervical Carotid Dissections with Endovascular Stents. Neurosurgery, 44, 755-761. https://doi.org/10.1097/00006123-199904000-00037

[8] Tsuboki, S., Kawano, T., Ohmori, Y., Amadatsu, T. and Mukasa, A. (2019) Surgical Treatment of Spontaneous Internal Carotid Artery Dissection with Abducent Nerve Palsy: Case Report and Review of Literature. World Neurosurgery, 125, 10-14. https://doi.org/10.1016/j.wneu.2019.01.096

[9] Schievink, W.I., Piepgras, D.G. and McCaffrey, T.V. (1994) Surgical Treatment of Extracranial Internal Carotid Artery Dissecting Aneurysms. Neurosurgery, 35, 809-816. https://doi.org/10.1227/00006123-199411000-00002

[10] Liu, A.Y., Paulsen, R.D., Marcellus, M.L., et al. (1999) Long-Term Outcomes after Carotid Stent Placement for Treatment of Carotid Artery Dissection. Neurosurgery, 
45, 1368-1374. https://doi.org/10.1097/00006123-199912000-00022

[11] Touze, E., 11.Meary, E., Arquizan, C., et al. (2000) Aneurysmal Forms of Cervical Artery Dissections: Risk Factors and Prognosis. Neurology, 54, A378.

[12] Schievink, W.I. (2000) The Treatment of Spontaneous Carotid and Vertebral Artery Dissections. Current Opinion in Cardiology, 5, 316-321.

https://doi.org/10.1097/00001573-200009000-00002 